\title{
Stress- and Time-Dependent Formation of Self-Lubricating In Situ Carbon (SLIC) Films on Catalytically-Active Noble Alloys
}

\author{
MORGAN R. JONES, ${ }^{1}$ FRANK W. DELRIO, ${ }^{1}$ THOMAS E. BEECHEM,${ }^{1,2}$ \\ ANTHONY E. MCDONALD, ${ }^{1}$ TOMAS F. BABUSKA,,${ }^{1,3}$ \\ MICHAEL T. DUGGER, ${ }^{1}$ MICHAEL CHANDROSS,${ }^{1}$ NICOLAS ARGIBAY,${ }^{1}$ \\ and JOHN F. CURRY (1) ${ }^{1,4}$ \\ 1.-Material, Physical, and Chemical Sciences Center, Sandia National \\ Laboratories, Albuquerque, NM 87123, USA. 2.-Center for Integrated \\ Nanotechnologies, Sandia National Laboratories, Albuquerque, NM 87123, USA. \\ 3.-Department of Mechanical Engineering, Florida State University, Tallahassee, FL, USA. \\ 4.-e-mail: jcurry@sandia.gov
}

Low shear strength (30 $\mathrm{MPa}$ ) organic films were grown in situ on $\mathrm{Pt}_{0.9} \mathrm{Au}_{0.1}$ surfaces via cyclic sliding contact in dry $\mathrm{N}_{2}$ with trace concentrations of ambient hydrocarbons. We present a systematic investigation of the stressand time-dependent film formation. Steady-state friction coefficients were found to be as low as $\mu \sim 0.015$ and inversely proportional to contact pressure, revealing non-Amontonian behavior. Above a Hertzian contact pressure of $\sim 500 \mathrm{MPa}$, shear strength dropped, indicating an activated process. Raman spectroscopy identified non-uniformity in areal coverage and relative order with contact pressure. Regions of steady-state low-friction behavior exhibited spectra similar to DLC coatings. Atomic force microscopy was used to study the formation and growth of films at the nanoscale. Stress- and time-dependent measurements suggested a sublinear increase of film volume with time, and a transition from growth to wear at a Hertzian contact pressure of $\sim 1.2$ GPa.

\section{INTRODUCTION}

Investigations of organic film formation on catalytic metal surfaces began as early as 1958 with Hermance and Egan's work on mated palladium sliding contacts in vapor or liquid hydrocarbon environments. ${ }^{1}$ They believed that chemisorption of organics on a catalytic metal surface, followed by frictional activation and polymerization, was responsible for the visible accumulation of a dark brown solid surface film. Subsequent characterization showed that the film was an amorphous, high molecular weight material, that they referred to as a "frictional polymer." Although studies of contact force-dependent and speed-dependent formation were carried out, the mechanisms of formation were not clearly defined. Many studies thereafter sought to explore the possibility of using this frictional polymer as a means to realize self-lubrication and wear reduction in specialized situations, such as sliding in inaccessible enclosures under conditions where conventional lubrication was not feasible. ${ }^{2-5}$

Chaikin's work on frictional polymer formation attempted to better understand film formation though the lens of catalysis and charged particle emission. ${ }^{6}$ Although this study showed that electrostatic interactions played a role, it was noted that catalyst inhibitors were inconsequential in the production of frictional polymers. So, although catalysis is a popular explanation for tribopolymer generation, the interplay of catalysis, mechanochemistry, and electrostatic interactions remain incompletely understood. There is consensus, however, that the mechanisms for forming a frictional polymer in situ require at least three conditions: the presence of organics, a catalytically-active substrate, and shear between surfaces (i.e., sliding contacts). ${ }^{4}$ 
Meeting each of these "necessary conditions," multiple routes for the formation of carbonaceous self-replenishing films on metallic surfaces have been identified. $^{7-13}$ The goal has been to realize selflubricating films with characteristics similar to traditional carbon-containing coatings [e.g., graphitic or diamond-like carbon (DLC) films] that have been used for decades to reduce friction and wear. ${ }^{14}$ Recently, Argibay et al. ${ }^{15}$ found what is likely the first in situ formation of carbonaceous films on $\mathrm{Pt}_{0.9} \mathrm{Au}_{0.1}$ alloys at room temperature with friction coefficients $(\mu<0.02)$ similar to solid lubricants (i.e., DLC and $\mathrm{MoS}_{2}$ ). The nanocrystalline $\mathrm{Pt}_{0.9} \mathrm{Au}_{0.1}$ alloy (hereafter termed a Pt-Au thin film) used in this study is likely catalytically-active while exhibiting high hardness $(\sim 7 \mathrm{GPa})$ and exceptional wear resistance. ${ }^{16}$ The formation of these highly lubricious tribofilms at low temperature from ambient hydrocarbon species is novel and of significant practical interest, given the simple requirements to activate the process. Owing to the promise of these Pt-Au films and the uncertainty of frictional film formation in general, we investigate here how contact pressure, environment, and the number of sliding cycles affects the structural and tribological properties. Specifically, we present the results of a systematic investigation of formation rate, composition, and structure of these in situ films, with the aim of better understanding the stress and time dependencies of their formation. The tribological performance of the carbonaceous tribofilms is examined, and the structure of the films are characterized using Raman spectroscopy. The formation and growth of the tribofilms are studied at the nanoscale using atomic force microscopy. We show that, under the contact pressures employed, particularly at higher pressures, the tribofilms have shear strengths and structural characteristics like those of traditional DLC coatings.

\section{MATERIALS AND METHODS}

\section{Macroscale Tribology Experiments}

Macroscale tribological experiments were performed to investigate the formation of carbonaceous tribofilms on Pt-Au-coated steel substrates in a dry $\mathrm{N}_{2}$ environment. Material synthesis and characterization of the as-deposited Pt-Au substrates were described previously. ${ }^{15,16}$ Six different contact forces (presented throughout as contact pressures calculated by assuming a Hertzian contact) were used to investigate stress-dependent behavior: 10 $\mathrm{mN}(0.25 \mathrm{GPa}), 50 \mathrm{mN}(0.43 \mathrm{GPa}), 100 \mathrm{mN}(0.55$ $\mathrm{GPa}), 200 \mathrm{mN}(0.67 \mathrm{GPa}), 400 \mathrm{mN}(0.85 \mathrm{GPa})$, and $500 \mathrm{mN}(0.94 \mathrm{GPa})$. Under each loading condition, a 3.2-mm-diameter sapphire sphere was run in a bidirectional sliding motion against a Pt-Au-coated steel coupon. All experiments were run with a 1$\mathrm{mm} / \mathrm{s}$ sliding speed over a 2-mm track length (with the exception of the stripe test, described below), while normal and frictional force data was acquired at $500 \mathrm{~Hz}$. Experiments were run for 2000 bidirectional sliding cycles, except for the $0.94 \mathrm{GPa}$ test, which was run for 3500 cycles to verify the steadystate behavior. To compare the results with standard DLC coatings, experiments were also run on hydrogenated amorphous carbon coatings doped with silicon (a-C:H:Si) on 440C steel coupons under the same contact conditions above, but for only a subset of contact pressures $(0.43 \mathrm{GPa}, 0.55 \mathrm{GPa}$, and $0.94 \mathrm{GPa}$ ). Three replicate experiments were performed for each contact condition mentioned above, with a new sapphire sphere used for each test. The tribometer was previously described in Argibay et al., ${ }^{15}$ and was housed in an acrylic environmental chamber that was purged with ultrahigh purity $\mathrm{N}_{2}$ at $34 \mathrm{~L} / \mathrm{min}$ until less than $20 \mathrm{ppm}$ of $\mathrm{O}_{2}$ and $\mathrm{H}_{2} \mathrm{O}$ were present. No hydrocarbon species were intentionally introduced into the environmental enclosure during testing. It is likely that ambient hydrocarbons are solely responsible for the formation of the tribofilm, ${ }^{15}$ and as such were not controlled or measured. Throughout the experiment, $\mathrm{O}_{2}$ levels were continuously measured using an Alpha Omega 3000 series oxygen analyzer. Samples were rinsed in IPA/methanol prior to the first wear series on each sample.

An additional macroscale tribological experiment was performed to enable more detailed analyses and comparison of the cycle-dependent formation behavior of the tribofilms generated on Pt-Au surfaces. To accomplish this, a stripe test ${ }^{17}$ was performed where the number of cycles increased as the track length decreased at predetermined cycle intervals. Under a constant contact pressure of $0.55 \mathrm{GPa}$, the track length (beginning at $4 \mathrm{~mm}$ ) decreased linearly by $1 \mathrm{~mm}$ as the sliding cycle number increased by a factor of 10 , up to a maximum of $10^{3}$ bidirectional sliding cycles, so that segments at least $1 \mathrm{~mm}$ long were available for subsequent characterization.

\section{Nanoscale Tribology Experiments}

Nanoscale tribology experiments were performed on an Asylum MFP-3D atomic force microscope (AFM). Pt-Au-coated silicon substrates were fixed to AFM sample pucks and mounted inside the closed fluid cell on the sample stage. The fluid cell was attached directly to high-purity $\mathrm{N}_{2}$ through a flow meter, such that the cell maintained positive pressure and the relative humidity was close to that of the dry $\mathrm{N}_{2}$ source $(<20 \mathrm{ppm})$. Rectangular Si cantilevers with polycrystalline diamond coatings on the tip side and reflective aluminum coatings on the detector side were utilized to facilitate high wear resistance and enhance laser reflectivity (Nanosensors DT-CONTR tips with a nominal spring constant, $k_{\mathrm{c}}=0.5 \mathrm{~N} / \mathrm{m}$ ). The actual $k_{\mathrm{c}}$ value of each cantilever was measured via the thermal fluctuation method, ${ }^{18}$ and the resulting $k_{\mathrm{c}}$ values were within the range specified by the manufacturer. In addition, each cantilever was inspected before and after each test using scanning electron 
microscopy (SEM) to assess the diameter and to check for damage or material transfer. Tip diameters ranged in size from $280 \mathrm{~nm}$ to $320 \mathrm{~nm}$ and no changes in shape or size were observed after testing. The nanoscale growth and wear experiments consisted of a two-step, repeating process: (1) a contact-mode AFM image over a $1 \mu \mathrm{m} \times 1 \mu \mathrm{m}$ area (64 pixels $\times 64$ pixels) at a scan speed of $50 \mu \mathrm{m} / \mathrm{s}$ to enable tribofilm growth or wear, and (2) an intermittent-contact (i.e., tapping) mode AFM image over a $3 \mu \mathrm{m} \times 3 \mu \mathrm{m}$ area (512 pixels $\times 512$ pixels) at a scan speed of $7.5 \mu \mathrm{m} / \mathrm{s}$ to assess the tribofilm volume $(V)$ and morphology. While resulting in contact pressures higher than the macroscale experiments, the normal forces used were limited by the stiffness of the cantilever, and varied from $2 \mathrm{nN}$ to $120 \mathrm{nN}$, consistent with maximum contact pressures of $0.8 \mathrm{GPa}$ to $3.1 \mathrm{GPa}$ from Hertzian contact mechanics. ${ }^{19}$ The overall procedure was similar to that developed in Gosvami et al. ${ }^{20}$

\section{Raman Spectroscopy and Imaging}

Raman spectroscopy and imaging were conducted on a WiTec alpha 300R confocal Raman system employing a 532-nm incident laser focused through an objective with a $\times 50$ magnification and a 0.55 numerical aperture. A power of $5 \mathrm{~mW}$ was incident on the sample. No changes in the optical image or spectra after laser exposure were observed at this power level. Unpolarized light was collected and dispersed using a $600-1 / \mathrm{mm}$ grating that, in combination with an Andor CCD camera, resulted in a spectral accuracy of $\sim 1 \mathrm{~cm}^{-1}$. Raman images were acquired over varying areas with a Raman spectrum acquired every $2 \mu \mathrm{m}$. Carbon concentration was estimated by quantifying the strength of the Gmode near $1580 \mathrm{~cm}^{-1}$ relative to the baseline of the signal near $2000 \mathrm{~cm}^{-1}$. All spectral fitting was performed using a Britt-Wigner-Fano (BWF) lineshape in accordance with Ferrari and Robertson. ${ }^{21}$

\section{RESULTS}

\section{Stress-Dependent Tribofilm Formation}

Under all load conditions at the macroscale, the system initially exhibited a friction coefficient of $\mu \sim$ 0.35 , in agreement with previous experiments with sapphire on Pt-Au thin films in ambient laboratory air. ${ }^{15}$ After a run-in period, however, the formation of a carbonaceous tribofilm led to a significantly lower steady-state friction coefficient $(\mu<0.02)$. The $0.94 \mathrm{GPa}$ contact pressure produced the lowest friction, with an average steady-state friction coefficient of $\mu \sim 0.016$, as shown in Fig. 1. The highest steady-state friction was $\mu \sim 0.13$, corresponding to the $0.25 \mathrm{GPa}$ contact pressure. The results suggest that even in an inert, dry environment, the trace amounts of ambient hydrocarbons (i.e., parts per billion) in the environmental enclosure-as well as any latent carbon on the Pt-Au surface ${ }^{22}$-led to the formation of lubricious carbon-based tribofilms.
The steady-state friction coefficient was plotted against inverse Hertzian contact pressure to determine if the shear strength of the in situ tribofilm was similar to plasma-enhanced chemical vapor deposition (PECVD) DLC films and composites. It is well known that within the elastic regime, the coefficient of friction of solid lubricant films like DLCs is inversely proportional to contact pressure, ${ }^{23-25}$ and the relationship $\mu=\tau_{0} / P+\alpha$ holds, where $\mu$ is the coefficient of friction, $\tau_{0}$ is the shear strength, $P$ is the Hertzian contact pressure, and $\alpha$ is the pressure coefficient of the interfacial shear strength, which has been found to be negligible for many materials, including solid lubricants and metals. ${ }^{19,26,27}$ Here, a linear regression was plotted through the datasets, and the slope was used to determine $\tau_{0}$ for each solid lubricant. ${ }^{27}$ For the films on Pt-Au, a notably higher value of $\mu$ was observed at the lowest contact pressures, resulting in a large increase in $\tau_{0}$. Mohrbacher and Celis observed a similar phenomenon with hydrogenated amorphous carbon coatings under similar contact pressures; ${ }^{28}$ they hypothesized that this deviation was attributed to a change in the surface structure and properties of the tribofilm. The transition they observed was also marked by the shift to a more negative $\alpha$ coefficient $(\alpha=-0.02$ to $\alpha=-0.23){ }^{28}$ Tribofilms formed on Pt-Au exhibited relatively low $\alpha$ coefficients $(\alpha=-0.012)$ by comparison but were higher than the PECVD-deposited DLC $(\alpha=-$ $0.006)$ and DLN films $(\alpha=-0.006)$ tested (Fig. 1). It is possible that these differences in $\alpha$ between PECVD-deposited films and tribofilms formed on Pt-Au are due to the increased influence of hydrocarbons on the Pt-Au surface or by the higher degrees of disorder in such an amorphous solid. ${ }^{29}$

Despite the increase at low contact pressures, the carbonaceous tribofilms exhibit a shear strength comparable to traditional lubricious films. This was deduced by comparing their response with two additional DLC films, namely a commercial DLC film formed via PECVD and a diamond-like nanocomposite (DLN; DLC doped with Si and O). ${ }^{30}$ The shear strength of the Pt-Au thin film DLC for low inverse contact stresses, between $1 \mathrm{GPa}^{-1}$ and 2 $\mathrm{GPa}^{-1}$, converged to a value of $\tau_{0} \sim 30 \mathrm{MPa}$ as compared to $15 \mathrm{MPa}$ and $9 \mathrm{MPa}$ for the DLC and DLN films, respectively. These comparatively small differences in shear strength between the differing films are not surprising, however, as film thickness, $\mathrm{sp}^{2} / \mathrm{sp}^{3}$ carbon content and structure, and the degree of hydrogenation or other compositing agents $^{31,32}$ each affect this value.

To further probe the contact stress-dependent formation of the carbonaceous film, Raman spectroscopy was used to characterize differences in the amount of carbonaceous material deposited and differences in its chemical structures. These results are displayed in Fig. 2 for three different applied pressures, for which optical images and Raman maps of G-mode $\left(\sim 1580 \mathrm{~cm}^{-1}\right)$ intensity were taken 


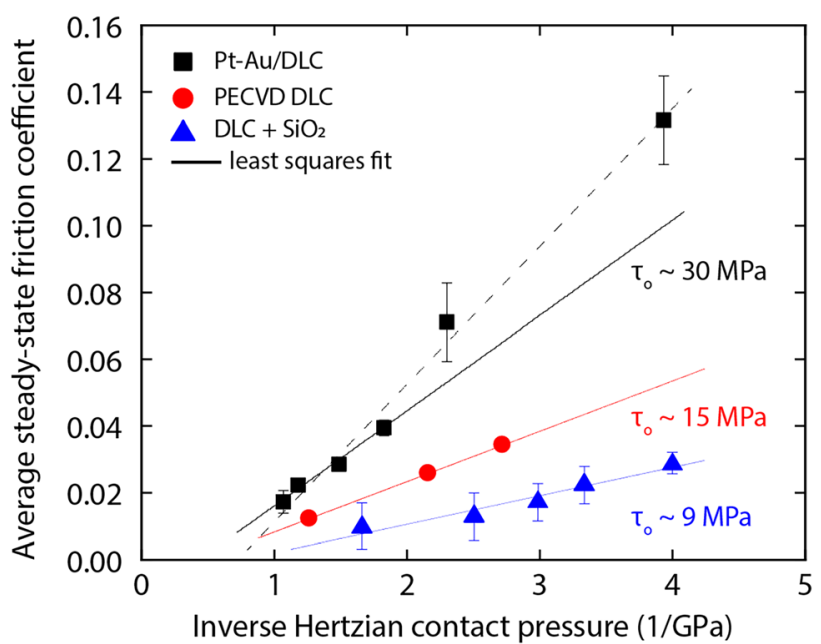

Fig. 1. Average steady-state $\mu$ as a function of inverse contact pressure $\left(\mathrm{GPa}^{-1}\right)$ run in a dry $\mathrm{N}_{2}$ environment for Pt-Au/DLC tribofilms (black squares), PECVD DLC films (red circles) and DLN films $\left(\mathrm{DLC}+\mathrm{SiO}_{2}\right)$ (blue triangles).30 A linear regression was plotted through each dataset to determine the corresponding values for $\tau_{0}$. Uncertainty intervals in reported friction coefficient correspond to the standard deviation for each test in the steady-state regime. For the $\mathrm{Pt}-\mathrm{Au} / \mathrm{DLC}$ data the black dotted regression line encompasses all contact pressure conditions, whereas the black solid regression line excludes the $3.93 \mathrm{GPa}^{-1}(0.25 \mathrm{GPa})$ and $2.3 \mathrm{GPa}^{-1}(0.43 \mathrm{GPa})$ conditions that exhibited higher friction (Color figure online).

to qualitatively assess the carbon coverage on the $0.94 \mathrm{GPa}, 0.43 \mathrm{GPa}$, and $0.25 \mathrm{GPa}$ tracks. Averaged Raman spectra were taken along an $\sim 5 \mu \mathrm{m}$ wide region along the center of the wear scars (referred to as centerline measurements) (Fig. 2d), as well as offcenterline measurements that encompassed the rest of the wear scar outside the centerline (Fig. 2e). These results show an increased amount of carbonaceous film on the Pt-Au with increasing contact pressure. Beyond the amount of carbon deposited, Raman also indicates differences in the order of the resulting carbon in the tribofilm, as well as slight differences between the material generated along the track centerline (the location with peak Hertzian contact pressure) versus the rest of the contact. For Raman spectra taken along the track centerline (Fig. 2d), the highest contact pressure $(0.94 \mathrm{GPa})$ resulted in a slightly more disordered $\mathrm{sp}^{2}$ carbon signal. This was deduced by observing a small downshift in the G-mode $\left(\sim 1588 \mathrm{~cm}^{-1}\right.$ to $\left.1581 \mathrm{~cm}^{-1}\right)$ peak position in conjunction with a slight asymmetric broadening of peaks $\left(\mathrm{Q}^{-1} ;-6.8\right.$ to -4.3 (Table I)). ${ }^{21}$ Spectra acquired outside the track centerline (Fig. 2e) showed smaller differences, but similar qualitative characteristics, in that slightly more disorder was found with larger contact pressures. These differences in both the amount of carbon and their order qualitatively correlate with the steadystate friction coefficients.

Owing to this link between performance and film growth, the stress-dependent growth kinetics were also examined via nanoscale tribological experiments with AFM, as shown in Fig. 3. In this series of experiments, contact-mode scans were generated at contact pressures ranging from $0.8 \mathrm{GPa}$ to 3.1 $\mathrm{GPa}$, and intermittent-contact mode images were taken at 500-cycle increments up to a total of 4500 cycles. Figure $3 a$ shows intermittent-contact mode images for a 4000-cycle region as a function of pressure, and Fig. 3b presents the resulting tribofilm volume (in and out of the contact region) for multiple cycle numbers (up to 4000 cycles) as a function of contact pressure. The data suggest that the growth kinetics exhibited two separate regimes as a function of contact pressure. At lower contact pressures (1.2 $\mathrm{GPa}$ and below), total tribofilm volume $(V)$ appears to increase linearly with contact pressure with tribofilm growth occurring vertically and laterally, but only inside the contact region. At $1.2 \mathrm{GPa}$, signs of tribofilm wear begin to occur, as growth at the center of the contact region recedes, and deposits at the edges begin to accumulate. At contact pressures above $1.2 \mathrm{GPa}$, the tribofilm within the contact region was completely displaced by the AFM tip, appearing to expose the Pt-Au substrate (Fig. 3a). Interestingly, the increase in tribofilm volume as a function of contact pressure slowed considerably at the onset of wear $(1.2 \mathrm{GPa})$, yet grew considerably at the highest contact pressure tested (3.1 GPa). It may be that renewed contact of the Pt-Au substrate with the AFM probe in addition to higher contact pressures accelerated chain scission of adsorbed organics, ${ }^{15}$ leading to this enhanced growth.

\section{Time-Dependent Tribofilm Formation}

To tie the changes in friction over time explicitly to changes in structure and coverage, a macroscale stripe test was performed at a contact pressure of $0.55 \mathrm{GPa}$. Using the same sapphire sphere, a test was run for 1 cycle over $4 \mathrm{~mm}$, 10 cycles over $3 \mathrm{~mm}$, 100 cycles over $2 \mathrm{~mm}$, and 1000 cycles over $1 \mathrm{~mm}$. Raman spectroscopy (Fig. 4) was utilized in each stripe segment to probe the carbon content as a function of total cycles (i.e., 1/10/100/1000 cycles). Figure $4 a-d$ shows carbon signal maps taken from the centerline of the wear track from each segment. As the number of cycles increased, the carbon signal and carbon coverage increased, with brighter regions containing more carbon and likely thicker deposits. Figure $4 \mathrm{e}$ shows the carbon signal and $\mu$ as a function of the number of cycles. The highest carbon signal, and lowest friction coefficient, were observed at 1000 cycles. Spectra for each segment (Fig. 4f) show the transition from a low-intensity amorphous carbon signal for the very first bidirectional pass to a spectrum representative of increased order within the carbon film, with discernible separation of the D- and G-mode peaks at 100 cycles to 1000 cycles. D-mode and G-mode peak positions, as well as $\mathrm{Q}^{-1}$, for the Raman results in Fig. 4, are provided in Table II, where order in the carbon nominally increases with number of cycles. 

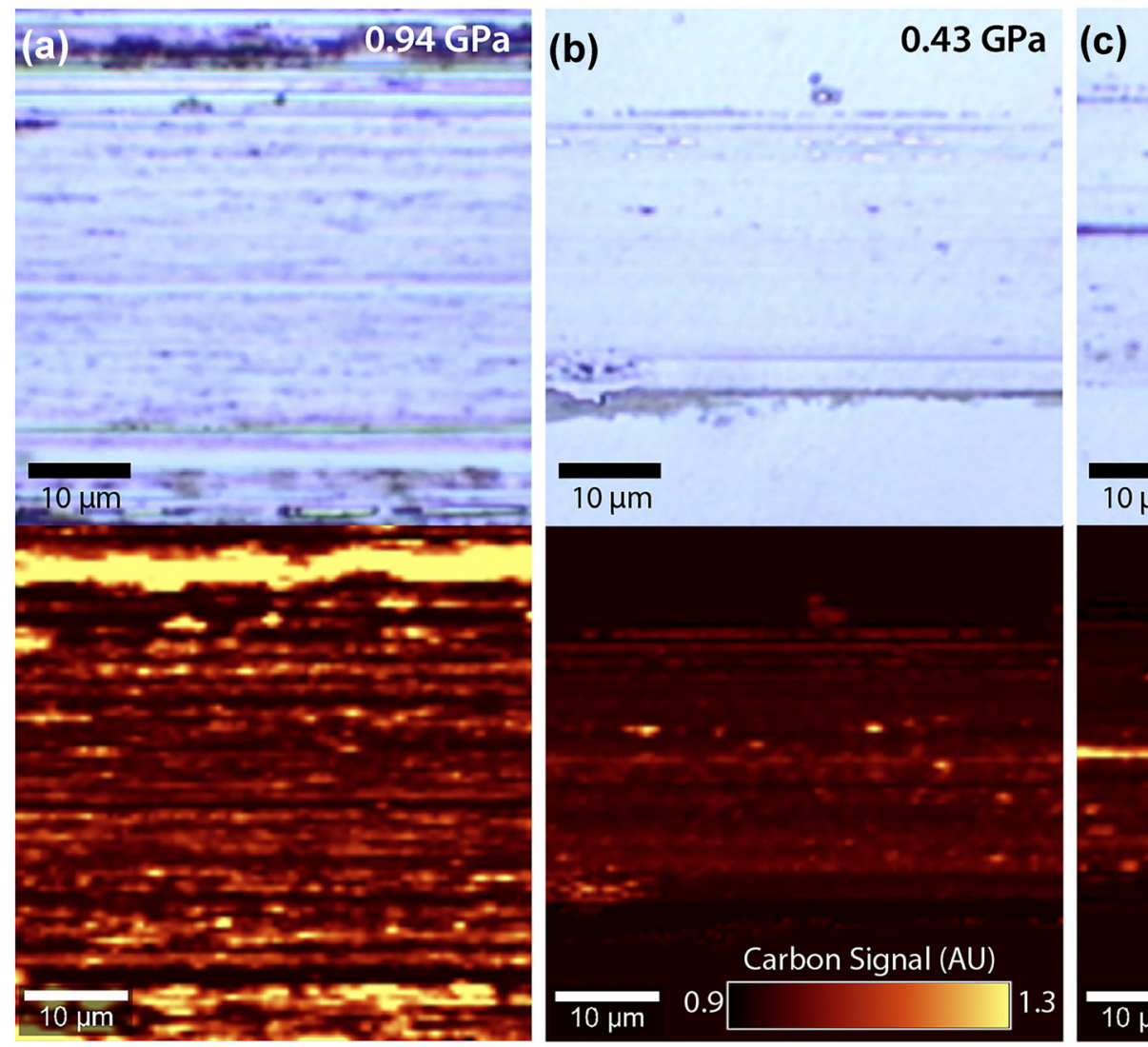

$0.25 \mathrm{GPa}$
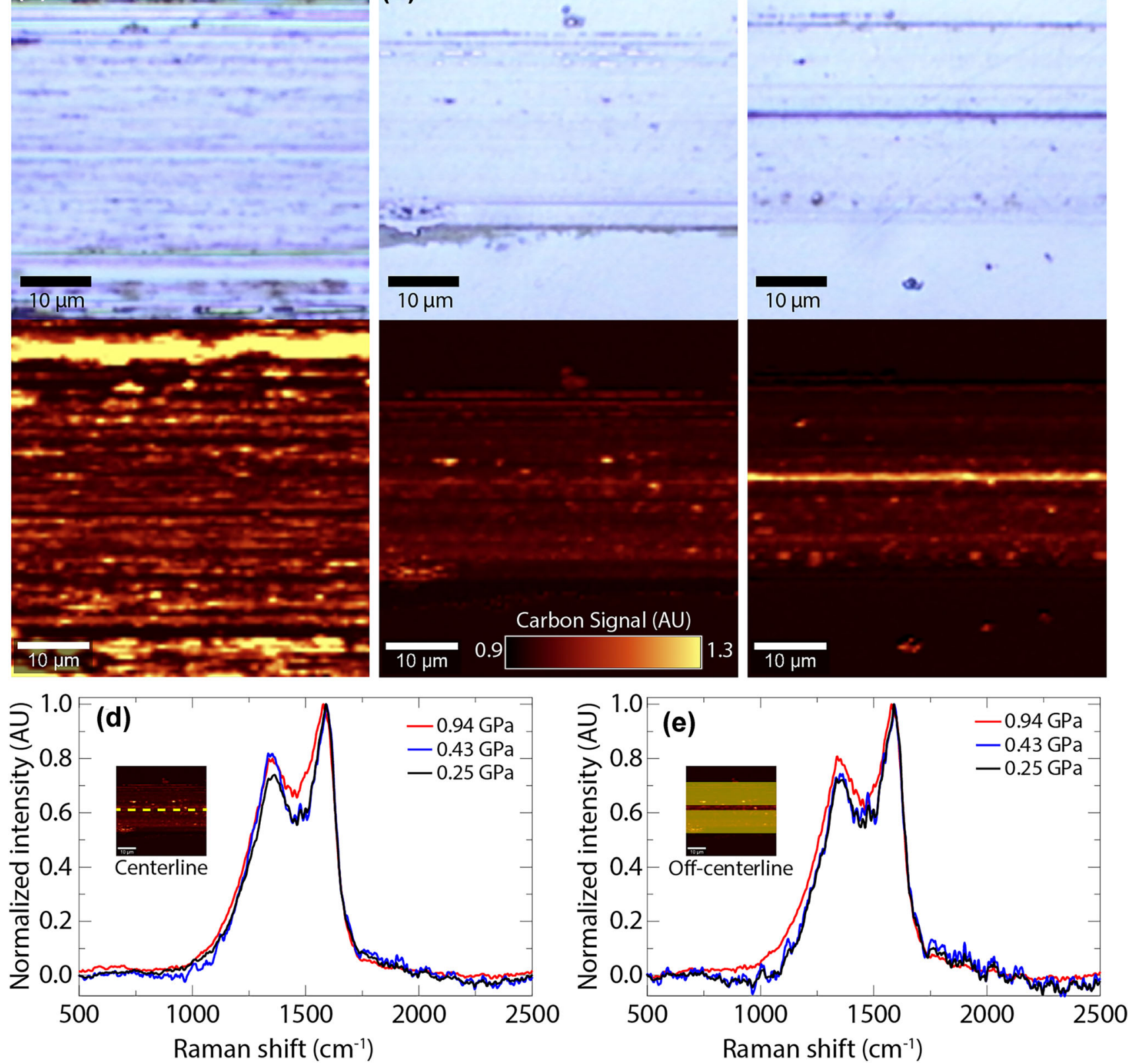

Fig. 2. Optical images of wear scars and Raman spectra maps of G-mode intensity for (a) $0.94 \mathrm{GPa}$, (b) $0.43 \mathrm{GPa}$, and (c) $0.25 \mathrm{GPa}$ contact pressures. Average spectra for tribofilms along (d) the track centerline and (e) the off-centerline, showing a general increase in tribofilm formation (brighter regions signifying more carbon signal), as well as an increase in disorder at the peak Hertzian contact pressure along the track centerline, as evident by a downshift in G-peak position and asymmetric broadening of the D- and G-mode peaks.

The time-dependent growth kinetics were further examined from nanoscale tribology experiments with AFM, as shown in Fig. 5. The results in Fig. 5 are a recast version of the same results from Fig. 3, where contact-mode regions were generated at contact pressures ranging from $0.8 \mathrm{GPa}$ to 3.1 $\mathrm{GPa}$, and intermittent-contact mode images were taken at 500-cycle increments for 4500 total cycles. Figure 5a shows intermittent-contact mode images for a contact pressure of $1.2 \mathrm{GPa}$ as a function of number of cycles, while Fig. $5 \mathrm{~b}$ presents the tribofilm volume for contact pressures ranging from $0.8 \mathrm{GPa}$ to $3.1 \mathrm{GPa}$ as a function of number of cycles. Figure 5a shows that the tribofilm exhibited patchy growth in the contact region. Previous work has suggested that film nucleation and growth may be dependent on surface heterogeneities, such as defects and roughness, that translate into heterogeneities in film thickness. ${ }^{20}$ From the data in Fig. 5b, it is also evident that tribofilm volume asymptotically approached a steady-state value for all $P$, suggesting that the efficacy of the catalytic 
Table I. G-mode $\left(\omega_{G}\right)$ and D-mode $\left(\omega_{D}\right)$ peak positions together with coupling coefficients from BWF lineshape fits as a function of maximum Hertzian contact pressure and wear track position, derived from spectra in Fig. 2.

\begin{tabular}{|c|c|c|c|c|}
\hline Max Hertzian contact pressure (GPa) & Track position & $\omega_{G}\left(\mathrm{~cm}^{-1}\right)$ & $\omega_{D}\left(\mathrm{~cm}^{-1}\right)$ & $Q^{-1}$ \\
\hline 0.94 & Center & 1581 & 1348 & -4.3 \\
\hline 0.43 & Center & 1589 & 1346 & -6.9 \\
\hline 0.25 & Center & 1588 & 1354 & -6.8 \\
\hline 0.94 & Off-center & 1584 & 1349 & -5.4 \\
\hline 0.43 & Off-center & 1586 & 1355 & -8.9 \\
\hline 0.25 & Off-center & 1587 & 1354 & -8.1 \\
\hline
\end{tabular}
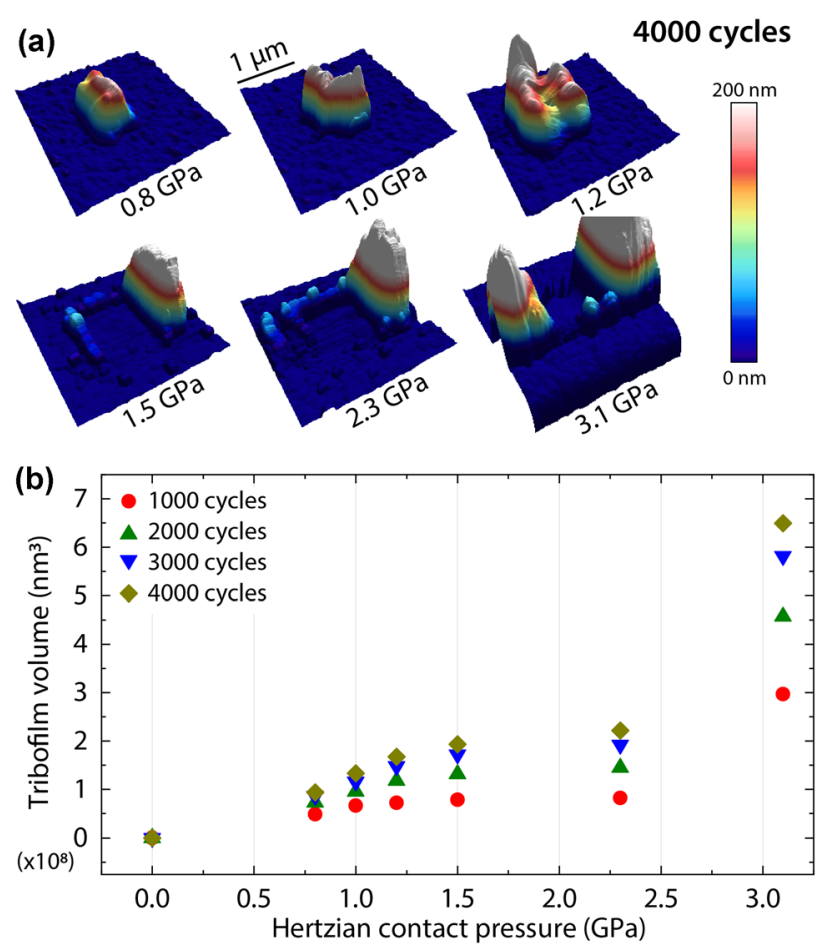

Fig. 3. Tribofilm formation as a function of contact pressure as determined from the nanoscale experiments. (a) Intermittent-contact mode topography images ( $3 \mu \mathrm{m} \times 3 \mu \mathrm{m}$ area) of the contact region after 4000 cycles at contact pressures of $0.8 \mathrm{GPa}, 1.0 \mathrm{GPa}, 1.2 \mathrm{GPa}$, $1.5 \mathrm{GPa}, 2.3 \mathrm{GPa}$, and 3.1 GPa. (b) Tribofilm volume as a function of contact pressure for experiments up to 4000 cycles.

process that allows film growth decreased as the number of cycles increased. Finally, it is important to note that the average film thickness from Fig. 5b $(\sim 100 \mathrm{~nm}$, assuming a representative volume of $\sim$ $10^{8} \mathrm{~nm}^{3}$ over an AFM contact area of $10^{6} \mathrm{~nm}^{2}$, or $1 \mu \mathrm{m}^{2}$ ) was consistent with thickness measurements in macroscale wear tracks ( $~ 50 \mathrm{~nm}$ to 200 $\mathrm{nm}$, from Argibay et al. ${ }^{15}$ ), implying that the growth kinetics are dependent on contact pressure and not probe diameter.

\section{DISCUSSION}

From the stress-dependent friction coefficient measurements (Fig. 1), the tribofilms formed in situ on Pt-Au were found to have shear strength (30 $\mathrm{MPa})$ and behaviors similar to that of sliding pairs involving DLC films and composites. ${ }^{24,28,30,33,34}$ For example, Cui et al. reported a shear strength of $\tau_{0} \sim 16 \mathrm{MPa}$ for hydrogenated amorphous DLC films in a dry $\mathrm{N}_{2}$ environment, ${ }^{33}$ and Zhao et al. reported a shear strength of $\tau_{0} \sim 10$ MPa for Ti-DLC films in ambient air. ${ }^{34}$ As reported previously, ${ }^{15}$ similar tribofilms formed on Pt-Au exhibited a relatively low degree of hydrogenation $(20 \%)$ as well as Pt-Au nanoparticles dispersed throughout the thickness of the film. This may explain the slightly higher shear strengths observed here as compared to pure DLC tribopairs. More interestingly, steady-state $\mu$ results as a function of inverse Hertzian contact pressure suggest experiments run at lower contact pressures exhibit higher shear strengths (Fig. 1). Mohrbacher and Celis reported similar behavior in hydrogenated amorphous carbon films run in air with $5 \%$ relative humidity. ${ }^{28}$ It is possible that this threshold was observable for Mohrbacher and Celis due to the effects of non-carbonaceous adsorbates (water or oxygen) disrupting either transfer and/or running film formation at lower contact pressures. ${ }^{35}$ Disruption or thinning of transfer films in DLC contacts is known to result in friction instabilities or film failure, ${ }^{36,37}$ which may also explain the higher shear strengths observed here at lower contact pressures. Raman maps of film formation as a function of contact pressure (Fig. 2a-c) and sliding cycles (Fig. 4a-d) suggest that an increase in tribofilm coverage and thickness promotes low and consistent friction behavior. Interestingly, at the comparable contact pressures tested in both the macroscale (Fig. 2a) and nanoscale (Fig. 3a) experiments, the tribofilm coverage/thickness was much greater at the edges of contact as compared to the track centerline or the region in contact in AFM. This behavior is similar to transfer films formed in DLC tribopairs, where others have hypothesized that, unlike the base DLC coating, transfer films formed in the contact are more polymeric in nature. As such, the tribofilms exhibit viscoelastic properties that allow them to squeeze outside the nominal contact area, like what we observe. ${ }^{38,39}$

In addition to highlighting the changes in coverage and thickness/intensity of carbon in wear scars, Raman spectroscopic maps of tribofilms were useful 

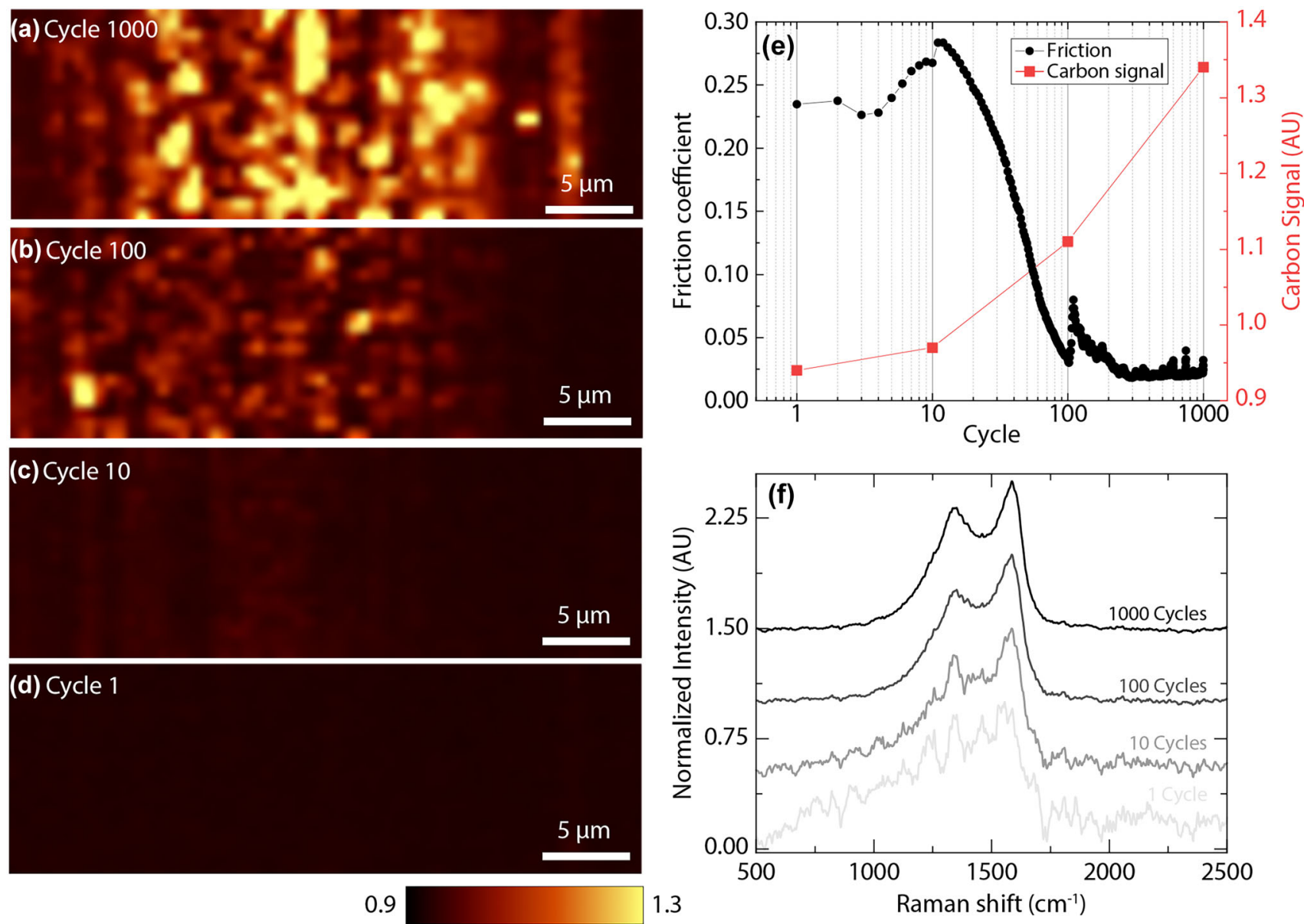

0.9

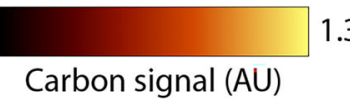

Fig. 4. Raman spectra maps of G-mode intensity on a stripe test under a constant 0.55-GPa contact pressure at (a) 1000, (b) 100, (c) 10, and (d) 1 bi-directional sliding cycles. A plot of (e) carbon signal (red squares) and friction coefficient (black circles) as a function of cycle number, as well as (f) averaged Raman spectra for each cycle segment, show that the increasing number of cycles led to stronger and more ordered carbon signals, as well as higher surface coverage and decreasing friction coefficients (Color figure online).

Table II. G-mode $\left(\omega_{G}\right)$ and D-mode $\left(\omega_{D}\right)$ peak positions together with coupling coefficients from BWF lineshape fits as a function of maximum Hertzian contact pressure and sliding cycles, derived from spectra in Fig. 4.

\begin{tabular}{|c|c|c|c|c|}
\hline Max Hertzian contact pressure (GPa) & Sliding cycles & $\omega_{\mathbf{G}}\left(\mathbf{c m}^{-1}\right)$ & $\omega_{\mathbf{D}}\left(\mathbf{c m}^{-1}\right)$ & $Q^{-1}$ \\
\hline 0.43 & 1 & 1567 & 1325 & -3.4 \\
\hline 0.43 & 10 & 1583 & 1346 & -4.0 \\
\hline 0.43 & 100 & 1582 & 1349 & -4.3 \\
\hline 0.43 & 1000 & 1586 & 1344 & -4.5 \\
\hline
\end{tabular}

in assessing if contact pressure or time in contact influenced the structure of these carbonaceous deposits in any meaningful way. Works from Ferrari, Casiraghi, and Robertson provide a means of interpreting visible Raman spectra to characterize amorphous carbon films, via the $\mathrm{sp}^{2}$ carbon bonding characteristics manifesting in the shape and intensity of the D- and G-mode peaks..$^{21,40,41}$ In general, the average Raman spectra taken as a function of both contact pressure and cycle count shown here were remarkably similar to transfer films formed when sliding against DLC coatings. As others have shown, the Raman spectra for these transfer films are quite distinct from the base DLC coating, and typically exhibit a comparatively higher degree of ordering. ${ }^{36,37,42-44}$ This increase in ordering was most evident for in situ-generated tribofilms formed on Pt-Au substrates as a function of increasing cycle count (Fig. 4). Maps and average Raman spectra taken at lower cycle counts indicated the formation of a low intensity amorphous carbon material, while higher cycle counts gradually showed the 

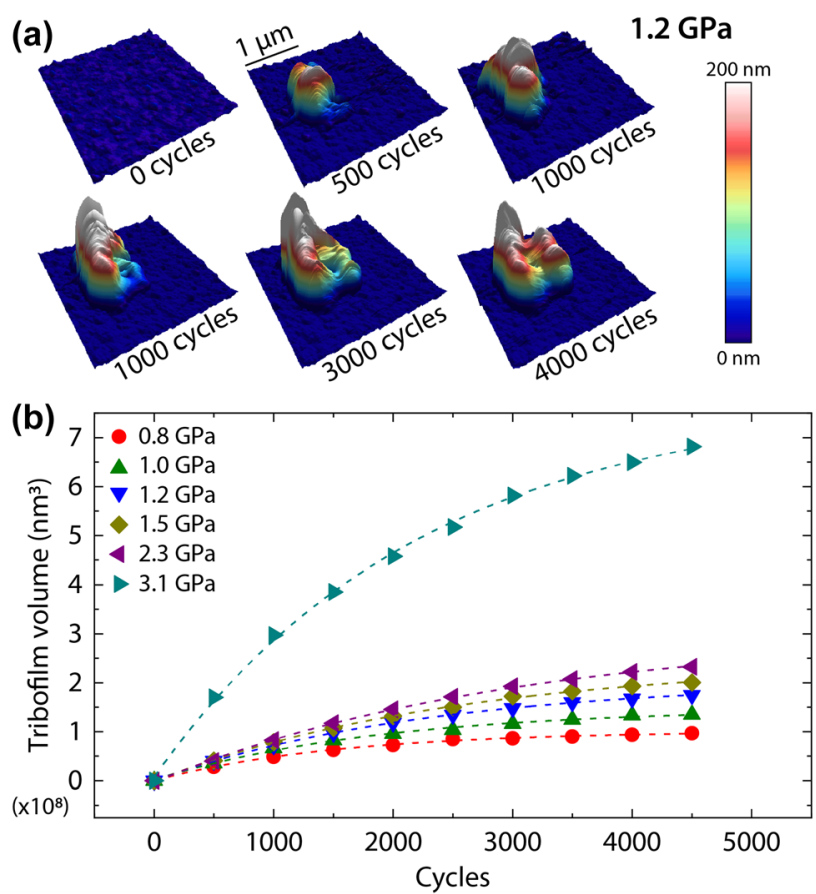

Fig. 5. Tribofilm formation as a function of number of cycles as determined from the nanoscale experiments. (a) Intermittent-contact mode topography images ( $3 \mu \mathrm{m} \times 3 \mu \mathrm{m}$ area) of the contact region at a contact pressure of $1.2 \mathrm{GPa}$ after 0 cycles, 500 cycles, 1000 cycles, 2000 cycles, 3000 cycles, and 4000 cycles. (b) Tribofilm volume as a function of cycles for contact pressures up to $3.1 \mathrm{GPa}$. For all $P$, tribofilm volume increased asymptotically to a steady-state value at large numbers of cycles.

emergence of distinct and prominent D- and G-mode peaks. The 100- and 1000-cycle stripe segments were structurally very similar to one another (Table II), and exhibited the lowest friction coefficients in the stripe test. Similarly, the more disordered material at shorter cycle counts exhibited higher friction, suggesting a general decrease in friction with an increase in ordering of the tribofilm.

In contrast, tribofilms generated on Pt-Au in the macroscale experiments exhibited a slight increase in disorder of the carbon structure with increasing contact pressure and subsequently decreasing friction coefficients (Fig. 2). As mentioned earlier, this slight decrease in ordering at the highest contact pressures $(0.94 \mathrm{GPa})$ is signified by a downshift in the G-mode $\left(\sim 1580 \mathrm{~cm}^{-1}\right)$ peak position, as well as a slight asymmetric broadening between peaks (Table I). ${ }^{21}$ This observed increase in disorder is relatively small compared to lower loads, and is only observed in the centerline of the wear track where the peak Hertzian contact pressure is highest. It is also interesting that, at the lowest contact pressure $(0.25 \mathrm{GPa})$, the tribofilm is concentrated at the track centerline (Fig. 2c), and ultimately accelerates in growth rate and begins to pile up at the edges of the wear scar (Fig. 2a) at the highest contact pressure $(0.94 \mathrm{GPa})$. A similar trend is seen in the AFM results (Fig. 3), where tribofilm volume generally increases with contact pressure across two distinct regimes. Below the $1.5 \mathrm{GPa}$ contact pressures used in AFM, growth of an adherent tribofilm occurs within the contact region, similar to what is observed at the track centerline under the peak Hertzian contact pressure of $0.25 \mathrm{GPa}$ in macroscale experiments (Fig. 2(c)). One possible explanation for this relatively steady rate of increase in tribofilm formation at lower contact pressures may be related to the contact area. Hermance and Egan's work on tribopolymer formation suggested that the increase in contact area as a function of increasing force may explain the increase in tribopolymer yield as a function of load, as it enables the incorporation of more organics into the tribopolymer formed through

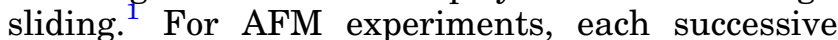
pass of the AFM probe within the rastered contact region contributes to the aggregate increase in tribofilm volume with increased contact area. At $1.5 \mathrm{GPa}$ contact pressures and above in AFM, growth of the tribofilm proceeds initially at a slower rate and wear of the tribofilm results in nearly all the volume being moved to the periphery of the contact, possibly from exceeding the adhesive strength of the tribofilms on Pt-Au. Again, this is similar to what is observed at the highest contact pressures assessed in macroscale experiments (Fig. 2a; $0.94 \mathrm{GPa}$ ) where the highest intensities of carbon signal are at wear scar edges. As is seen in Raman spectroscopy of macroscale experiments, a carbonaceous tribofilm still persists within the contact region, yet appears to be completely removed in nanoscale experiments at $1.5 \mathrm{GPa}$ and above. Despite this, growth of the tribofilm persists in nanoscale experiments at the higher contact pressures, likely driven by continuous replenishment of ambient organics at the surface that feed the formation before the film is pushed out of the contact region. It is also possible that the degree of reactivity of the diamond probes used in AFM differs from that of sapphire used in macroscale studies, as well as differences in shear rate, both of which are outside the scope of the current study.

At the highest contact pressure tested in AFM, $3.1 \mathrm{GPa}$, tribofilm growth increases dramatically (Fig. 3), but is still relegated to the periphery of the contact area. Such an increase in growth rate relative to lower contact pressures suggests the activation of a distinct formation mechanism. At higher contact pressures, greater formation is likely stimulated by more intimate contact with the catalytic Pt-Au substrate, as well as a higher degree of chain scission of organic molecules on the surface. ${ }^{15}$ More chain scission of adsorbed organics on the surface would likely lead to more disordered carbon networks on the surface, as well as more undercoordinated carbons available for passivation and bonding to grow the tribofilm. Raman spectroscopy on the macroscale wear scar at $0.94 \mathrm{GPa}$ (Fig. 2d) corroborates this proposed mechanism, showing an increased disorder of the carbon in the 
tribofilm with contact pressure. No evidence of wear of the Pt-Au substrate was observed for the AFM studies, even at the highest contact pressures tested. This may be due to the continuous adsorption and degradation of a hydrocarbons producing a sacrificial film. Also, the relatively high hardness of Pt-Au $(\sim 7 \mathrm{GPa})$ could be a contributing factor to the observed wear resistance during testing. ${ }^{16}$

Recent studies by Gosvami et al. ${ }^{20}$ noted the growth and wear regions during similar singleasperity tribofilm growth studies with zinc dialkyldithiophosphate (ZDDP). Interestingly, the transition between tribofilm growth and wear for ZDDP-based tribofilms was $\sim 5 \mathrm{GPa}$ (compared to $\sim$ $1.2 \mathrm{GPa}$ for our tribofilms), suggesting more wear resistant films or a difference in film growth mechanisms and adhesion to the substrate. Debris generation outside the contact region at higher contact pressures was also not observed, possibly due to dispersal in the liquid medium used for testing. Our time-dependent measurements (Fig. 5), though, indicate some significant differences in the reaction pathways. For our tribofilms formed on Pt-Au, the volume asymptotically approached a steady-state value for all pressures, suggesting that the efficacy of the catalytic process decreased as the number of cycles increased. In contrast, the ZDDP-based tribofilms showed an initial linear and subsequent power-law growth, consistent with a zero-order and $n$ th-order $(n=0.22)$ reaction, respectively, as cycles increased. Curiously, the films formed from ZDDP additives grew on various substrates, while these lubricious tribofilms likely depend on the catalytic Pt-Au thin films, and this may be the basis for the different reaction pathways. Additional studies controlling the composition, structure, and concentration of organics in the environment will be critical in fully understanding tribofilm formation mechanisms on Pt-Au surfaces.

\section{SUMMARY AND CONCLUSION}

A systematic study on catalytically-active noble alloy surfaces was carried out to examine the stressand time-dependent formation of extremely lowfriction, self-lubricating in situ carbon tribofilms. In dry $\mathrm{N}_{2}$ sliding conditions, we have shown that carbonaceous tribofilms readily form on $\mathrm{Pt}-\mathrm{Au}$ thin films, resulting in steady-state friction coefficients as low as $\mu \sim 0.016$ at a maximum Hertzian contact pressure of about $1 \mathrm{GPa}$. The friction coefficient of these tribofilms showed a clear contact stress dependence similar to the behavior of commercial DLC films. The shear strength of the tribofilm was $\tau_{0} \sim 30 \mathrm{MPa}$, slightly higher than those of DLCs in the literature. ${ }^{24,28,30,33,34}$ Raman spectroscopy demonstrated that an increase in contact pressure led to an increase in coverage, concentration, and disorder of the tribofilm. AFM experiments confirmed the increase in surface coverage (volume) with pressure, but also highlighted a transition from film growth to wear at a threshold contact pressure near $1.2 \mathrm{GPa}$. Stripe tests at constant pressure showed time-dependent formation of the tribofilm, with carbon concentration and degree of ordering of the amorphous carbon structure increasing with cycle count (time). Finally, time-dependent AFM experiments demonstrated a sublinear increase in film volume with time, suggesting that the efficacy of the catalytic process decreased as the number of cycles increased.

\section{ACKNOWLEDGEMENTS}

This work was undertaken, in part, at the Center for Integrated Nanotechnologies (CINT), an Office of Science User Facility operated for the US Department of Energy (DOE) Office of Science.This work was funded by the Laboratory Directed Research and Development program at Sandia National Laboratories, a multi-mission laboratory managed and operated by National Technology and Engineering Solutions of Sandia, LLC., a wholly owned subsidiary of Honeywell International, Inc., for the U.S. Department of Energy's National Nuclear Security Administration under contract DENA0003525. Any subjective views or opinions that might be expressed in the paper do not necessarily represent the views of the U.S. Department of Energy or the United States Government.

\section{CONFLICT OF INTEREST}

The authors declare that they have no conflict of interest.

\section{OPEN ACCESS}

This article is licensed under a Creative Commons Attribution 4.0 International License, which permits use, sharing, adaptation, distribution and reproduction in any medium or format, as long as you give appropriate credit to the original author(s) and the source, provide a link to the Creative Commons licence, and indicate if changes were made. The images or other third party material in this article are included in the article's Creative Commons licence, unless indicated otherwise in a credit line to the material. If material is not included in the article's Creative Commons licence and your intended use is not permitted by statutory regulation or exceeds the permitted use, you will need to obtain permission directly from the copyright holder. To view a copy of this licence, visit $h$ ttp://creativecommons.org/licenses/by/4.0/.

\section{REFERENCES}

1. H.W. Hermance and T.F. Egan, Bell Syst. Tech. J. 37, 739. (1958).

2. W.E. Campbell and R.E. Lee, ASLE Trans. 5, 91. (1962).

3. M.J. Furey Wear 26, 369. (1973).

4. W. Crossland and P. Murphy, IEEE Trans. PartsHybrids Pack. 10, 64. (1974).

5. H.C. Stinton, H.A. Spikes, and A. Cameron, ASLE Trans. 25, 355. (1982). 
6. S.W. Chaikin, Wear 10, 49. (1967).

7. Y. Liao, R. Pourzal, M.A. Wimmer, J.J. Jacobs, A. Fischer, and L.D. Marks, Science 334, 1687. (2011).

8. M.A. Wimmer, M.P. Laurent, M.T. Mathew, C. Nagelli, Y. Liao, L.D. Marks, J.J. Jacobs, and A. Fischer, Wear 332-333, 643. (2015).

9. M.A. Wimmer, A. Fischer, R. Büscher, R. Pourzal, C. Sprecher, R. Hauert, and J.J. Jacobs, J. Orthop. Res. 28, 436. (2010).

10. J. Hesketh, M. Ward, D. Dowson, and A. Neville, Biomaterials 35, 2113. (2014).

11. A. Erdemir, G. Ramirez, O.L. Eryilmaz, B. Narayanan, Y. Liao, G. Kamath, and S.K.R.S. Sankaranarayanan, Nature 536, 67. (2016).

12. B. Johnson, H. Wu, M. Desanker, D. Pickens, Y. Wah, and C.Q. Jane, Tribol. Lett. 66, 1. (2018).

13. X. He, A.J. Barthel, and S.H. Kim, Surf. Sci. 648, 352 (2016).

14. H. Wu, A.M. Khan, B. Johnson, K. Sasikumar, Y.-W. Chung, and Q.J. Wang, A.C.S. Appl. Mater. Interfaces 11, 16139. (2019).

15. N. Argibay, T.F. Babuska, J.F. Curry, M.T. Dugger, P. Lu, D.P. Adams, B.L. Nation, B.L. Doyle, M. Pham, A. Pimentel, C. Mowry, A.R. Hinkle, and M. Chandross, Carbon 138, 61. (2018).

16. J.F. Curry, T.F. Babuska, T.A. Furnish, P. Lu, D.P. Adams, A.B. Kustas, B.L. Nation, M.T. Dugger, M. Chandross, B.G. Clark, B.L. Boyce, C.A. Schuh, and N. Argibay, Adv. Mater. 30, e1802026. (2018)

17. K.L. Harris, A.A. Pitenis, W.G. Sawyer, B.A. Krick, G.S Blackman, D.J. Kasprzak, and C.P. Junk, Macromolecules 48, 3739. (2015).

18. J.L. Hutter and J. Bechhoefer, Rev. Sci. Instrum. 64, 1868. (1993).

19. D. Tabor and F.P. Bowden, Br. J. Appl. Phys., 17, (1966),

20. N.N. Gosvami, J.A. Bares, F. Mangolini, A.R. Konicek, D.G. Yablon, and R.W. Carpick, Science 348, 102. (2015).

21. A.C. Ferrari and J. Robertson, Phys. Rev. B 61, 14095. (2000).

22. P.J. Cumpson and M.P. Seah, Metrologia 33, 507. (2003).

23. A. Erdemir and C. Donnet, J. Phys. D 39, R311. (2006).
24. T.W. Scharf, J.A. Ohlhausen, D.R. Tallant, and S.V. Prasad, J. Appl. Phys. 101, 063521. (2007).

25. T.W. Scharf and I.L. Singer, Tribol. Trans. 45, 363. (2002).

26. P.W. Bridgman, Phys. Rev. 48, 825. (1935).

27. I.L. Singer, R.N. Bolster, J. Wegand, S. Fayeulle, and B.C. Stupp, Appl. Phys. Lett. 57, 995. (1990).

28. H. Mohrbacher and J.-P. Celis, Diam. Relat. Mater. 4, 1267. (1995).

29. G. He, M.H. Muser, and M.O. Robbins, Science 284, 1650. (1999).

30. T.W. Scharf and S.V. Prasad, J. Mater. Sci. 48, 511. (2013).

31. J. Robertson, Mater. Sci. Eng. R Rep. 37, 129. (2002).

32. H. Ronkainen, S. Varjus, J. Koskinen, and K. Holmberg, Wear 249, 260. (2001).

33. L. Cui, Z. Lu, and L. Wang, Tribol. Int. 82, 195. (2015).

34. F. Zhao, H. Li, L. Ji, Y. Wang, H. Zhou, and J. Chen, Diam. Relat. Mater. 19, 342. (2010).

35. M.J. Marino, E. Hsiao, L.C. Bradley, O.L. Eryilmaz, A. Erdemir, and S.H. Kim, Tribol. Lett. 42, 285. (2011).

36. T.W. Scharf and I.L. Singer, Tribol. Lett. 14, 137. (2003).

37. T.W. Scharf and I.L. Singer, Tribol. Lett. 14, 3. (2003).

38. J.B. McClimon, A.C. Lang, Z. Milne, N. Garabedian, A.C. Moore, J. Hilbert, F. Mangolini, J.R. Lukes, D.L. Burris, M.L. Taheri, J. Fontaine, and R.W. Carpick, Tribol. Lett. 67, 48. (2019).

39. X. Chen, T. Kato, and M. Nosaka, A.C.S. Appl. Mater. Interfaces 6, 13389. (2014).

40. C. Casiraghi, A.C. Ferrari, and J. Robertson, Phys. Rev. B 72, 085401. (2005).

41. A.C. Ferrari, and J. Robertson, Phys. Rev. B 64, 075414. (2001).

42. A. Erdemir, C. Bindal, J. Pagan, and P. Wilbur, Surf. Coat. Technol. 76, 559. (1995).

43. J.C. Sánchez-López, A. Erdemir, C. Donnet, and T.C. Rojas, Surf. Coat. Technol. 163, 444. (2003).

44. S. Liu, C. Zhang, E. Osman, X. Chen, T. Ma, Y. Hu, J. Luo, and E. Ali, Sci. China Tech. Sci. 59, 1795. (2016).

Publisher's Note Springer Nature remains neutral with regard to jurisdictional claims in published maps and institutional affiliations. 